\title{
Espaces de pacs : géographie d'une innovation sociale
}

\author{
Emmanuel Jaurand \\ Stéphane Leroy
}

EA 3482 Lab'Urba

Université Paris 12-Val-de-Marne, Créteil

\begin{abstract}
Résumé
En France, le pacte civil de solidarité (pacs) permet depuis 1999 l'union entre deux adultes quel que soit leur sexe. Porté par les gays et lesbiennes auxquels il offre une reconnaissance symbolique et un début d'égalité des droits en matière de vie de couple, il s'est progressivement banalisé, comme en témoigne son succès quantitatif croissant (plus de 200000 signatures en 6 ans). L'analyse de la géographie du pacs montre une diffusion à partir des capitales nationale et régionales vers tout le pays. Ce processus s'accompagne du maintien de singulières disparités spatiales à toutes les échelles, en particulier la place privilégiée de Paris et des Midis. Elles sont en partie explicables par le jeu de multiples facteurs sociaux, culturels et démographiques : il existe notamment une forte corrélation entre la concentration des professions intellectuelles du secteur public et le taux de pacs. La géographie de cette innovation sociale, incomplète en raison des lacunes de l'information (ignorance du sexe des pacsés), témoigne de la complexité des mutations actuelles de la société française, marquées notamment par l'émergence d'une nouvelle classe moyenne supérieure métropolitaine.
\end{abstract}

Mots-clés : pacs, France, innovation sociale, disparités spatiales, homosexuels.

\begin{abstract}
Since 1999, the civil solidarity pact ("pacs"), allows in France the union between two adults regardless of their sex. Supported by gays and lesbians to whom it gives a symbolic recognition and the beginning of rights equality in couple life matters, it progressively normalized itself as its increased success shows it (more than 200000 signatures in 6 years). The analysis of the geography of the "pacs" shows that it has spread from national and regional capitals toward all the country. This process took place with the upholding of remarkable spatial disparities at any scale, in particular the mean place of Paris and the South of France. We can partly explain them by the combination of a lot of social, cultural and demographic factors: there is a major correlation between the concentration of public creative professionals and the civil solidarity pact rate. The geography of this social innovation is uncompleted because of missing information (the sex of "pacs" signers is not recorded). It proves the complexity of the current social changes in France, characterized by the rise of a new metropolitan upper middle class.
\end{abstract}

Keywords : civil solidarity pact, France, social innovation, spatial disparities, homosexuals. 


\section{Introduction}

«Discipline menacée ou dominée, la géographie française a des raisons de développer une stratégie de citadelle assiégée, défendant ses positions et évitant de s'aventurer en terrain découvert (...)» (Collignon et Staszak, 2004, p. 39). Nous partageons ce constat. Encore trop peu de géographes français s'emparent des questionnements qui agitent la société, sans parler de la liste noire des sujets «tabous », laissant le champ libre aux historiens, sociologues ou anthropologues. Étudier la géographie du pacs, malgré le caractère imprécis des données, c'est postuler que l'analyse d'une pratique nouvelle est aussi intéressante en géographie que l'étude de macro-processus déjà bien connus; c'est aussi faire l'hypothèse que le pacs, dont l'adoption par le législateur fut compliquée (Encadré 1), participe des mutations actuelles de la société française et mieux, qu'il contribue à en expliquer la forme et la dimension spatiale.

\section{Encadré 1: \\ La difficile genèse du pacs}

Le 13 octobre 1999, l'Assemblée nationale adopte par 315 voix contre 249 une loi introduisant un nouveau titre dans le Code civil : "Du pacte civil de solidarité et du concubinage ». Le 15 novembre la loi est promulguée. Le pacs naît après plusieurs années de revendications associatives et de propositions de loi avortées, d'atermoiements et d'affrontements politiques.

Avec le pacs, deux personnes de sexe opposé mais aussi de même sexe peuvent organiser juridiquement leur vie de couple. "Ce n'est pas un progrès, comme on l'entend dire, car ce terme impliquerait l'inscription des unions homosexuelles sur une chaîne évolutive de civilisation contredite par l'observation ethnologique qui met sur le même plan toutes les formes sociales; c'est simplement l'actualisation nouvelle d'un possible jusqu'ici occulté » précise Françoise Héritier (2000, p. 478), opposée au pacs.

Engagées contre l'inégalité des droits selon l'orientation sexuelle, les associations gays et lesbiennes sont à l'origine de la loi (Schiltz, 2004), soutenues par quelques femmes et hommes politiques pour l'essentiel de gauche. C'est pourquoi, pour beaucoup des adversaires de la loi, le pacs est une sorte de mariage gay qui ne dit pas son nom.

Rarement sous la $V^{e}$ République, une proposition de loi n'a déclenché autant de débats et de commentaires, aussi bien dans la classe politique que dans l'opinion publique. Même la loi sur l'IVG de 1975 et celle sur l'abolition de la peine de mort de 1981 n'ont pas provoqué un débat d'une telle longueur (120 heures de discussions durant 13 mois, à partir d'octobre 1998) ni engendré une telle inflation de procédures (2 exceptions d'irrecevabilité, 3 navettes entre les deux chambres, 2000 amendements, la saisine du Conseil constitutionnel, etc.). Surtout, jamais une loi n'a entraîné le déferlement de tant de haine, dévoilant les sentiments homophobes d'une partie de la population, en particulier de plusieurs personnalités publiques de milieux très divers: politique, religieux, scientifique, médiatique, etc. Ils se sont opposés «à ce que le droit enregistre ce que le bon sens réprouve »(Eribon, 2000, p. 54). 
D'abord innovation juridique, le pacs peut-être considéré comme une innovation sociale dans la mesure où cette nouvelle pratique sociale a séduit plus de 400000 personnes au 31 décembre $2005^{1}$. L'une des raisons de l'intérêt que porte la géographie à l'innovation, qu'elle soit économique, sociale ou culturelle «réside dans la recherche de (ses) dimensions spatiales (...) »(Offner, 2003, p. 513), c'est-à-dire dans l'étude des modes et processus de sa diffusion spatiale, composante fondamentale de l'organisation et de l'évolution des territoires (Saint-Julien, 1995). Comme pour toutes les innovations, au moins à leur début, la géographie du pacs est celle d'un espace hétérogène et hiérarchisé, opposant les lieux qui ont déjà adopté l'innovation aux autres. Nous allons montrer que cette hiérarchie est relativement originale, mettant en évidence différents types d'interactions spatiales, et que le processus de diffusion spatiale du pacs est encore difficile à saisir. La recherche d'explications à ces configurations spatiales parfois inattendues impose de confronter la géographie du pacs à la géographie sociale de la France, au travers de différents indicateurs sociodémographiques (structure par âge, degré d'urbanisation, composition socioprofessionnelle, etc.).

Les députés P. Bloche et J.-P. Michel (2001) rappellent, dans leur rapport d'information à l'Assemblée Nationale, que: «Malgré le déchaînement homophobe qu'il suscita, le pacs a incontestablement permis de banaliser l'homosexualité, en l'intégrant dans un lien social clairement reconnu par le code civil ». Pourtant, de façon assez paradoxale, certains estiment que le pacs participe d'une institutionnalisation de la hiérarchie entre les sexualités (Rault, 2004). On peut tout de même penser que cette loi marque une réelle avancée en matière d'égalité des droits et de lutte contre les discriminations. Elle s'inscrit dans un mouvement plus général en France (lois sur la parité, le harcèlement sexuel, création de la Halde, etc.) et à l'échelle de l'Europe, dans laquelle des pays de plus en plus nombreux proposent un contrat d'union ouvert aux couples de même sexe (Encadré 2).

\section{Encadré 2 : \\ Les unions de couples de même sexe en Europe}

Parmi les pays européens qui reconnaissent les couples de même sexe, on trouve tous les pays scandinaves (le Danemark, pays pionnier, légalise les unions entre personnes de même sexe dès 1989), la Belgique, les Pays-Bas, l'Allemagne, la Hongrie et depuis peu le Royaume-Uni, la Suisse et la Slovénie. Si tous ces pays reconnaissent les unions homosexuelles, la forme de l'union et l'étendue des droits, entre le certificat d'union spécifique pour les gays et les lesbiennes et le mariage, comme en Belgique et aux Pays-Bas, avec ou sans droit à l'adoption, etc. demeurent très variables (Digoix et Festy, 2004 ; Festy, 2006).

Cette géographie européenne des droits des gays et lesbiennes qui opposa d'abord un nord "progressiste» et un sud "conservateur», a été bousculée par la décision du gouvernement socialiste espagnol d'ouvrir le mariage et le droit à l'adoption aux couples de même sexe en 2005. L'évolution de l'Espagne, pays fortement marqué par le catholicisme, montre que les sociétés occidentales sont en perpétuel mouvement, dessinant une géographie mouvante et de plus en plus difficile à saisir avec des méthodes, des modèles et des indicateurs « classiques ». 
Il convient toutefois d'insister sur le fait que le pacs ne concerne pas que la population homosexuelle, même si le débat s'est cristallisé sur ses droits et plus généralement sur sa visibilité (Digoix et Festy, 2004). Pour beaucoup de couples hétérosexuels, ce contrat représente aujourd'hui une alternative commode au mariage. Plus généralement, il répond à des attentes et des besoins divers de publics différents. Un an après l'entrée en vigueur de la loi, 70\% des Français interrogés l'approuvaient (Borrillo et Fassin, 2004). C'est pourquoi, après une année 2005 record (plus de 60000 pactes signés), on peut raisonnablement affirmer que le pacs est une réussite, mais une réussite délicate à étudier, en particulier dans sa dimension géographique.

\section{Le pacs : difficulté d'approche, incontestable succès}

Le profil des pacsés est inconnu, ce qui pourrait laisser croire à un «impossible bilan » (Festy, 2001, p. 1). Seule une approche quantitative globale est permise. Elle montre la réussite de cette innovation sociale.

\subsection{La géographie des pacsés n'est pas celle des homosexuels}

En 2006, aucune indication n'est disponible sur l'âge, la nationalité (alors que le pacs est possible entre un national et un étranger séjournant en France ou deux étrangers séjournant en France), la profession et catégorie socioprofessionnelle et sur le sexe des partenaires (Encadré 3). L'interdiction d'accès à ces données a été prononcée par la Commission Nationale Informatique et Libertés pour une raison officielle de protection de la vie privée. Cette louable intention ne cache-t-elle pas une certaine gêne par rapport à cette forme d'union, reléguée à un statut semi-clandestin ? Ou cela renvoie-t-il à un silence plus général sur certains critères de différenciation des personnes, considérés comme sensibles en France (religion et ethnicité par exemple) ? Le débat agite actuellement la communauté scientifique et divise la classe politique. Aux États-unis par exemple, le recensement comporte des rubriques sur les origines raciales comme sur le sexe des habitants d'un même logement. Même le poids relatif et la répartition des couples de même sexe sont connus, diffusés et cartographiés à différentes échelles (Coulmont, 2003 ; Gates et Ost, 2004). De même, dans plusieurs pays scandinaves des statistiques existent sur les contrats de partenariat réservés aux couples de même sexe (Festy, 2001).

En France, il s'avère impossible de connaître la proportion des unions de partenaires de sexe opposé et de même sexe, et à l'intérieur de ce dernier groupe, les parts respectives des couples féminins et masculins. Malgré la fin de la restriction sur la connaissance du sexe des pacsés (levée par la loi du 6 août 2004), le Ministère de la Justice a continué de fournir des statistiques muettes jusqu'en 2006 (Festy, 2006). A partir du $1^{\mathrm{er}}$ janvier 2007, sur décision de la Chancellerie, les greffes des tribunaux d'instance devront consigner le sexe des pacsés. Cette lacune de l'information, de 1999 jusqu'à aujourd'hui, n'interdit pas une évaluation globale mais fragilise toute interprétation fine des variations et des dynamiques spatiales du 
pacs. C'est pourquoi, une géographie quantitative des homosexuels demeure encore impossible en France.

\section{Encadré 3 : \\ Données et unités spatiales du pacs}

Les statistiques disponibles relatives aux contrats de pacs sont fournies par le Ministère de la Justice et publiées à l'issue de chaque trimestre. Elles portent sur les seuls éléments suivants: nombre de pacs signés (à multiplier par deux pour connaître le nombre de pacsés), nombre de refus et nombre de dissolutions. La signature du pacs se fait au greffe du tribunal d'instance du domicile commun ou de l'adresse de résidence choisie pour ceux qui n'habitent pas ensemble. Comme elle ne se fait pas en mairie, Wilfried Rault (2004) parle d'anomalie française à propos de ce choix et même de conditions humiliantes d'enregistrement. L'unité administrative de base des statistiques est donc celle du tribunal d'instance et non celle de la commune : on en compte 473 pour toute la France. La circonscription territoriale d'un tribunal d'instance couvre plusieurs cantons et a une taille généralement inférieure ou égale à celle d'un arrondissement. Des regroupements statistiques peuvent donc être opérés à l'échelle du département, de la circonscription d'une cour d'appel et de la région. Dans le cas exceptionnel de Paris, chacun des 20 arrondissements dispose d'un tribunal d'instance : il est donc permis pour cette seule commune de France de disposer d'informations à une échelle territoriale très grande; ce qui pourra faire l'objet d'un travail de recherche ultérieur.

Cependant une tendance, relative au sexe des pacsés et portant sur le premier mois d'existence du pacs est connue. En effet, la décision d'interdiction de la CNIL, adoptée par le Conseil d'État, n'est parue au Journal Officiel sous forme de décret que le 24 décembre 1999. Ainsi, le Président du «Collectif pour le PACS », Jean-Paul Pouliquen, après des enquêtes auprès de quelques greffes de tribunal d'instance a pu oser une estimation : «A Paris, $75 \%$ d'entre eux sont homosexuels, contre $40 \%$ seulement en province » (Holtz, 2000, p. 17). Tout récemment, le démographe Patrick Festy a estimé que les couples de même sexe représentaient la moitié des signataires juste après la création du pacs, sans toutefois préciser ses sources (Chayet, 2006).

S'il est difficile d'extrapoler à partir de ces connaissances discursives et indications statistiques partielles, on peut penser que les premières semaines du pacs ont connu un afflux de couples de même sexe dans les tribunaux d'instance, notamment à Paris où les gays sont certainement surreprésentés (Leroy, 2005), pour des raisons d'engagement militant et de reconnaissance sociale, puisque c'était la première fois qu'ils avaient la possibilité légale de faire reconnaître leur engagement commun. Daniel Borrillo et Eric Fassin (2004) considèrent même le pacs comme une sorte de seconde étape dans le processus de coming out (qu'on peut traduire par «sortie du placard $»^{2}$ ). D'ailleurs, dans les pays européens qui publient des statistiques sur les couples de même sexe, la première année a toujours été marquée par un 
nombre d'unions homosexuelles deux à trois fois plus important que les années suivantes (Festy, 2006). Il faut aussi rappeler le rôle des associations de défense des malades du sida (notamment Aides) dans la genèse du pacs (Eribon, 2003), car le texte prévoit notamment la possibilité d'une transmission du patrimoine ou, pour les locataires, du maintien dans les lieux du conjoint survivant. Le mariage restant réservé aux seules personnes de sexes différents, on peut s'attendre à ce que les homosexuels soient surreprésentés dans les signataires de pacs par rapport à leur poids dans la population. Cependant, des enquêtes informelles effectuées dans certains tribunaux d'instance montreraient une augmentation continue du nombre de contrats signés par des couples de sexe opposé; de fait, la part relative des pacs conclus entre personnes du même sexe aurait décliné depuis 2000 (Festy, 2006). Ce ne serait pas le moindre des paradoxes du pacs que d'avoir été réclamé par la «communauté » gay et lesbienne et stigmatisé pour cela par ses opposants, et de se trouver aujourd'hui conforté et plébiscité par les couples hétérosexuels.

\subsection{Le succès quantitatif croissant du pacs}

Les statistiques globales du nombre de pacs montrent un succès à la fois rapide et durable (Figure 1). Le démarrage a dépassé les prévisions les plus optimistes (Holtz, 2000), avec plus de 6100 pacs signés entre le 15 novembre et le 31 décembre 1999. Un rythme aussi soutenu ne se retrouve qu'à la fin de l'année 2004, et davantage encore au cours des deuxième, quatrième et surtout troisième trimestres de l'année 2005 (Figure 2). Cet enthousiasme initial se perpétue, certes de manière amoindrie, en 2000, avec un nombre de signatures de 22 276, supérieur à celui de l'année suivante, marquée par un creux (19 632).

Depuis le creux de 2001, le nombre de signatures de pacs s'est accru régulièrement jusqu'en 2004, sur un rythme annuel soutenu de 24 à $29 \%$. L'année 2005 est, elle, marquée par le nombre record de 60473 signatures, soit plus de 120000 pacsés, ce qui représente une augmentation de $50 \%$ par rapport au nombre de pactes signés en 2004 et équivaut à environ $15 \%$ d'une classe d'âges. Le total cumulé est ainsi de 205640 contrats au 31 décembre 2005 (12\% ont été dissous), ce qui représente un taux de 84,6 pacsés pour 10000 habitants de 18 ans et plus. Si les courbes du nombre de mariages et de pacs présentent des évolutions inverses depuis 6 ans, il reste que la baisse de la nuptialité est quasi-constante depuis une trentaine d'années (exception faite du «pic » de l'an 2000), et que le nombre de mariages est encore en 2005 près de cinq fois supérieur à celui des pacs ${ }^{3}$.

Ce succès incontestable montre clairement l'appropriation de ce nouveau contrat d'union par une partie de la société française. L'ancrage du pacs et l'accroissement quasi continuel du nombre de ses signataires signifient qu'il s'agit bien d'une innovation sociale et non d'une vogue passagère. «Les innovations ne se réduisent donc pas à des inventions et encore moins à des modes », comme le rappelle Thérèse Saint-Julien (1995, p. 559). Le pacs est mécaniquement plébiscité par ceux qui ne peuvent pas se marier. Il est aussi vraisemblablement adopté par ceux qui le peuvent mais ne le souhaitent pas, pour des raisons diverses : idéologiques, culturelles, symboliques ou simplement pratiques. Il est beaucoup 
plus facile et rapide à dissoudre que le mariage, tout en offrant quand même plusieurs de ses avantages. Son succès et le déclin relatif du mariage peuvent être en partie expliqués par ce que Daniel Borrillo et Eric Fassin appellent la logique du « démariage » (2004, p. 24), c'est-àdire une sorte de privatisation du mariage, qui n'est plus une obligation sociale mais seulement un choix personnel et intime. On peut rapprocher de cette logique de «réappropriation » du mariage par les individus, l'accroissement considérable du nombre de divorces : de 24 pour 100 mariages en 1980 à 42 pour 100 en 2003. D'une certaine manière, la recherche de la stabilité conjugale, imposée par les normes sociales, s'efface de plus en plus au profit de la libre expression des désirs et de la recherche de l'émancipation individuelle (De Singly, 2000). De même, à côté de l'effritement des obligations sociales, les croyances et les pratiques collectives déclinent. Robert Putnam (1990), à partir de l'exemple de l'évolution des adhésions aux clubs de bowling aux États-unis, montre la diminution de ce qu'il appelle le capital social et la transformation d'une société qui s'est construite avec des pratiques collectives à une société de d'individualisme et de l'entre-soi. Mais c'est aussi l'avènement d'une société «libérale», qui garantit et valorise la liberté de choix de chaque individu. Le pacs s'inscrit dans ces transformations de la famille et de la société.

Fig. 1 : Évolution du nombre de mariages et de pacs de 1999 à 2005

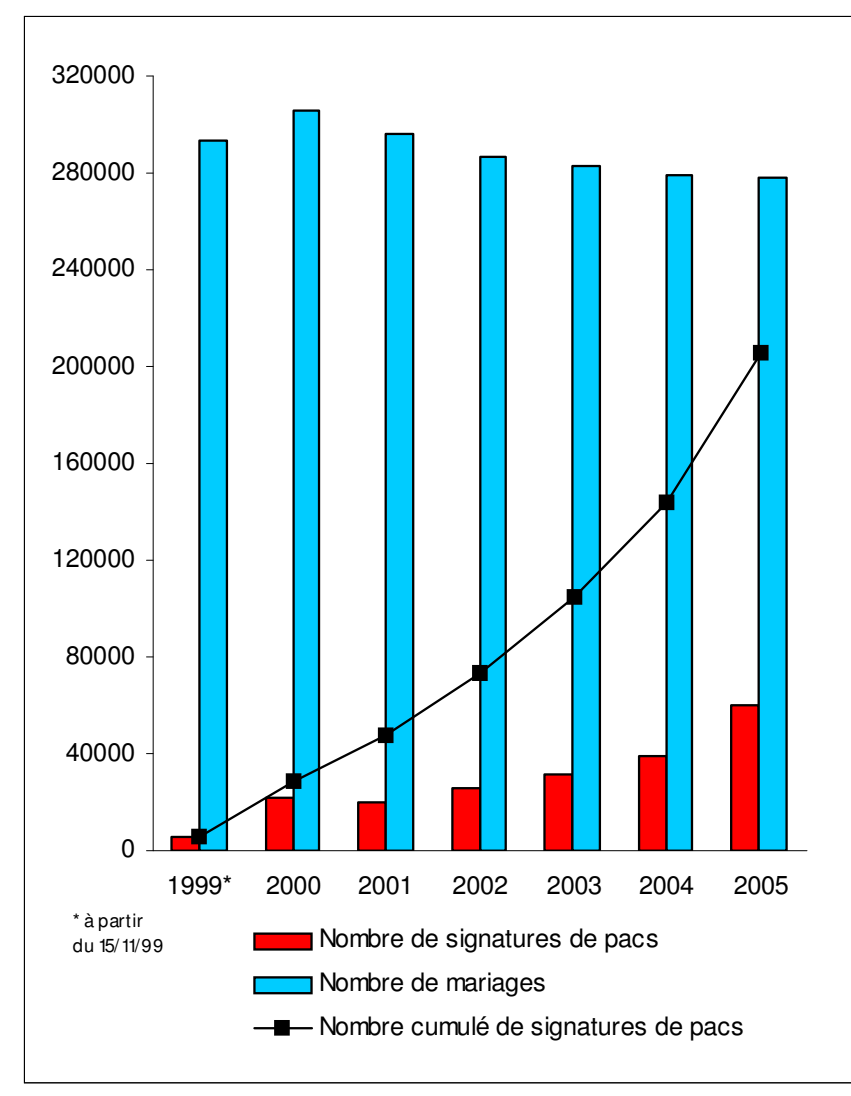

Sources : Ministère de la Justice ; INSEE.

Le rythme saisonnier du pacs privilégie les premier et dernier trimestres de l'année civile (Ruelland et Tonnerre, 2003), qui sont ceux de référence pour la déclaration fiscale 
commune (adresse du foyer au $1^{\mathrm{er}}$ janvier) et le rapprochement de conjoints dans la fonction publique (Figure 2). L'année 2005 bouleverse cette «règle », avec un pic record lors du troisième trimestre (plus de 20000 signatures), certainement lié à la décision du gouvernement, annoncée peu de temps auparavant, d'améliorer le dispositif du pacs.

Fig. 2 : Nombre de signatures de pacs par trimestre de 1999 à 2005

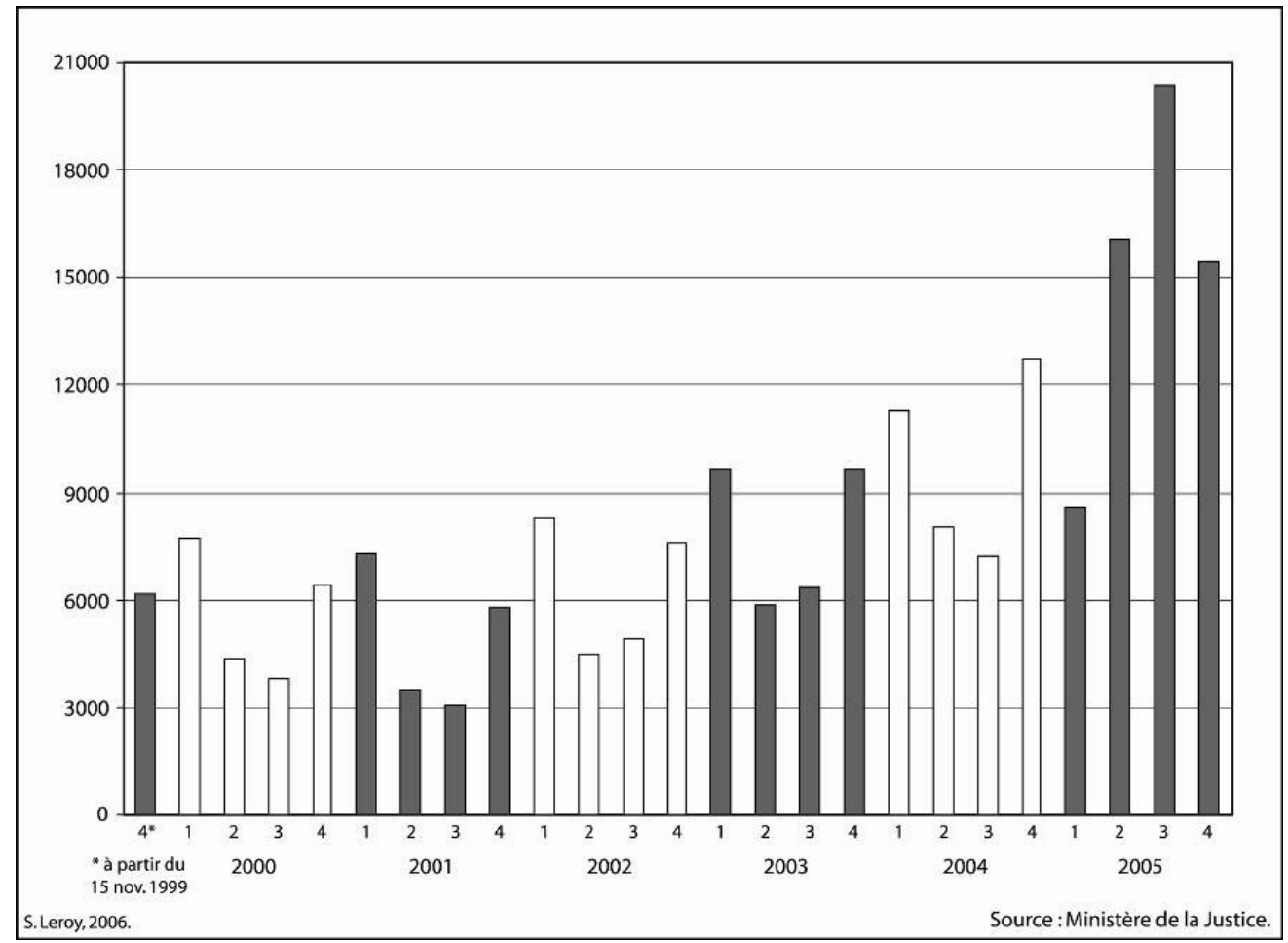

Au-delà de ces considérations, le flux quantitatif large et croissant des pacs nous semble avoir une signification socioculturelle voire politique. Nous n'hésitons pas à rapprocher son succès de la forte proportion de naissances hors-mariage (48,3\% en 2005 contre à peine plus de 10\% en 1980 : Richet-Mastain, 2006), qui fait de la France un des pays champions d'Europe en la matière, comme d'ailleurs pour le taux de fécondité, particulièrement élevé, ou encore la proportion de femmes de 50 ans ou plus qui ne se sont jamais mariées (Prioux, 2006). S'il est peut-être exagéré de parler de spécificité française, ces différents indicateurs montrent qu'une partie de plus en plus large de la société française s'écarte du modèle familial traditionnel, étant en cela certainement en avance sur une classe politique qui prétend souvent que «les Français ne sont pas prêts ». Revenue au pouvoir en 2002, la droite a d'ailleurs fait son mea culpa à propos du pacs : la loi de finances pour 2005 rapproche le régime fiscal des personnes pacsées de celui des couples mariés pour l'imposition commune et la donation, avec effet immédiat dès la signature du pacs. On peut s'interroger sur la signification de cette amélioration du pacs par ceux qui y étaient majoritairement opposés à l'origine. Est-ce le signe de l'acceptation de cette innovation sociale par le plus grand nombre ou un moyen pour rendre superflue la revendication du mariage pour les couples de même sexe? 


\section{Les espaces du pacs : des terres d'élection aux terres de mission}

Comme toute innovation sociale, le pacs est un révélateur de dynamiques sociospatiales à l'œuvre actuellement en France. Pour démêler l'écheveau de la géographie du pacs il est nécessaire de tester la pertinence de différents facteurs sociodémographiques. En fonction du niveau d'échelle et du maillage administratif retenu (région, département), des oppositions ou des rapprochements, tantôt bien connus, tantôt inattendus, apparaissent.

\subsection{Les Midis et l'Île-de-France en tête}

La carte du pacs à l'échelle régionale laisse apparaître de vastes ensembles homogènes (Figure 3). Si, avec un taux de 98,3 pacsés pour 10000 habitants de 18 ans et plus fin 2005, Midi-Pyrénées est la région qui arrive en tête, elle s'insère plus largement dans un arc des midis, de l'Aquitaine à Provence-Alpes-Côte-d'Azur, qui constitue avec Île-de-France (en seconde position dans la hiérarchie avec un taux de 97,6), l'ensemble régional le plus favorable au pacs. Dans ces 4 régions du sud de la France, le taux de pacsés s'élève à 93,1 pour 10000 habitants de 18 ans et plus. Toutes ces régions sont aussi celles où la part des pacs par rapport au nombre de mariages est parmi les plus élevées : en 2004, il s'est conclu 1 pacs pour 5 mariages en Midi-Pyrénées, 1 pour 6 en Aquitaine, contre 1 pour 10 dans le NordPas-de-Calais et 1 pour 11 en Corse.

Les régions d'élection du pacs ont finalement assez peu de caractères sociodémographiques identiques, hormis l'attractivité migratoire pour les jeunes adultes de leurs principaux pôles urbains (Léon et Godefroy, 2006) et une sous-représentation des ouvriers dans leur population active. Le coefficient de corrélation entre la part des ouvriers et celle des pacsés est égal à $-0,45^{4}$. Il y a manifestement une relation entre le niveau de qualification et l'importance relative du pacs $(r=0,44$ avec la part des cadres et professions intellectuelles supérieures et $r=0,63$ avec la part des diplômés de l'enseignement supérieur), même si bien d'autres facteurs jouent dans cette géographie: la Bretagne, la Corse et les DOM associent faibles proportions d'ouvriers et de pacsés.

Les régions les plus rétives au pacs sont bien loin de constituer un groupe homogène. Par ordre décroissant des taux de pacs, il s'agit de la région Centre, du Nord-Pas-de-Calais, de la Picardie et de la Corse. Celle-ci, avec un taux à peine supérieur à 50 pacsés pour 10000 habitants de 18 ans et plus, apparaît comme une région très hostile à cette forme d'union. Il faut ajouter à cette liste les quatre départements d'outre-mer. Toutes ces régions diffèrent par le degré d'urbanisation, la structure par âges, les activités économiques dominantes. Cependant, elles présentent certains traits sociodémographiques et culturels qui les rapprochent : une main-d'œuvre sous-qualifiée et une proportion de cadres plutôt faible. Il y a certainement d'autres facteurs explicatifs que ne saisissent pas les statistiques, facteurs d'ordre culturel tels que l'attachement aux valeurs familiales traditionnelles (Nord, Corse). Dans les DOM, fin 1999, aucune signature n'avait été enregistrée en Martinique, une seule en Guadeloupe, trois en Guyane et huit à la Réunion. Fin 2005, la Guyane affiche largement le 
meilleur taux de pacsés des DOM (71,6 pour 10000 habitants de 18 ans et plus), ce qui pourrait être lié à l'importance d'une population active qualifiée travaillant notamment dans le secteur aérospatial.

Fig. 3 : Répartition des signataires de pacs par région fin 2005

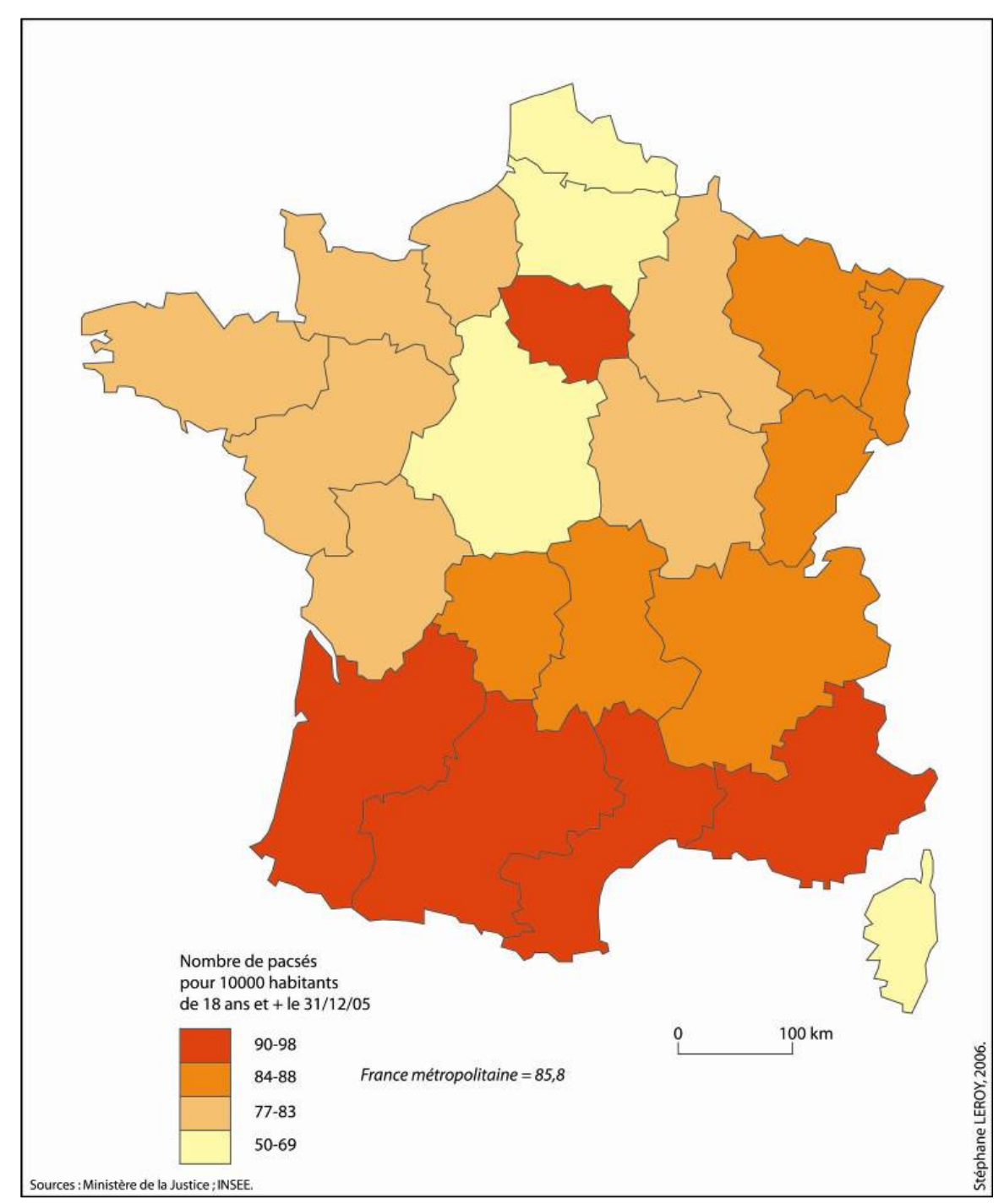

\subsection{L'avantage des capitales régionales et des villes universitaires}

Seuls 25 départements métropolitains ont un taux supérieur à la moyenne $(85,8)$ à la fin de l'année 2005. Les plus fortes proportions de pacsés se trouvent généralement mais pas systématiquement dans les départements les plus peuplés et urbanisés de chaque région (Figure 4). Les Alpes-de-Haute-Provence situées devant le Var, l'Isère devant le Rhône, les Pyrénées-Atlantiques devant la Gironde sont des exceptions. En revanche, dans la totalité des régions, les départements qui abritent la capitale ont un taux de pacs supérieur à celui de la moyenne régionale. De même, la part de ces départements dans le total des pacsés régionaux est systématiquement supérieure à leur importance démographique, à la seule exception près 
de la Lorraine (Figure 5). Le poids de ces départements est tel que dans 10 régions, ils abritent plus de la moitié du total des pacsés.

Fig. 4 : Répartition des signataires de pacs par département fin 2005

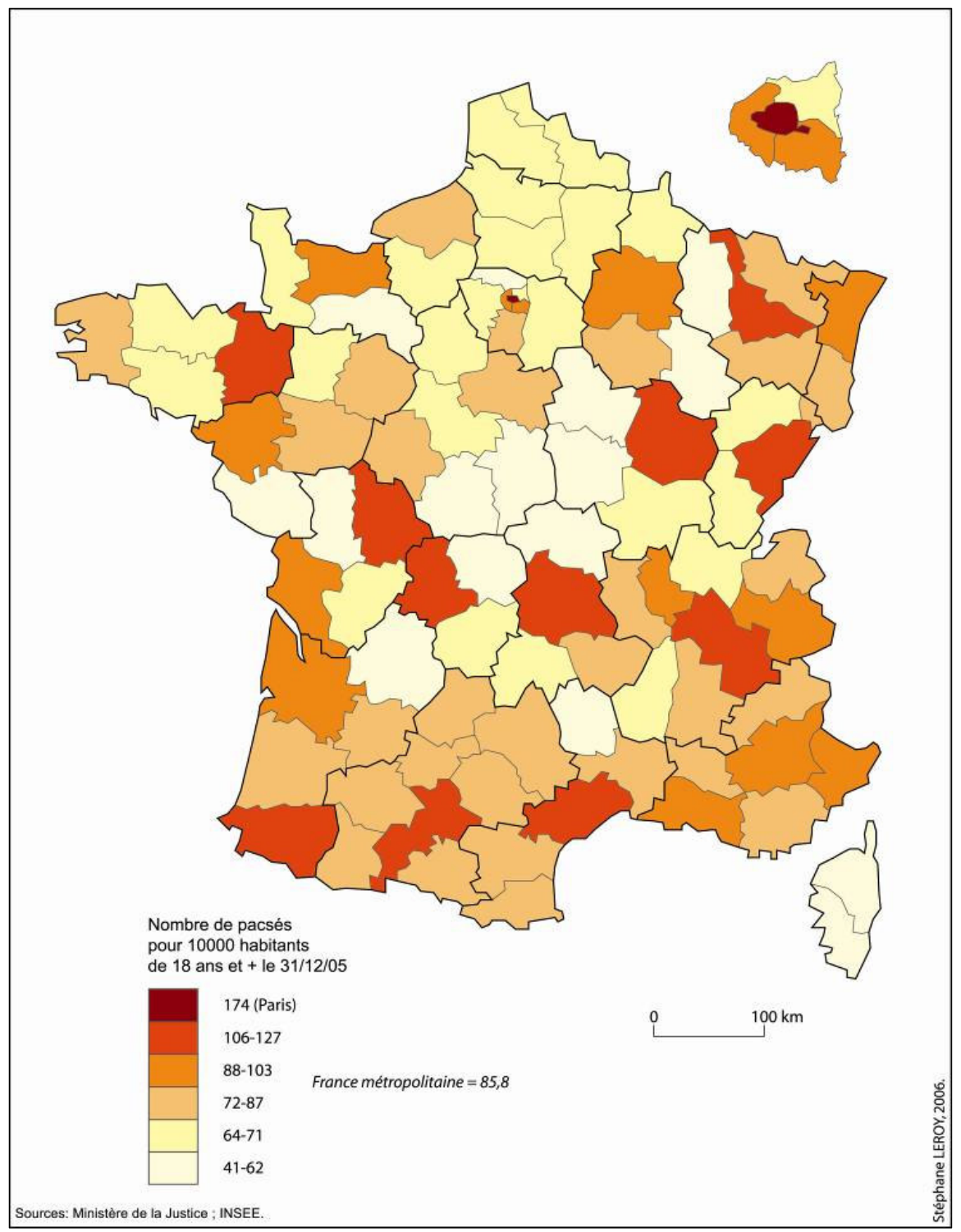

Ce clivage entre le centre et la périphérie des régions peut être analysé à une échelle plus fine que celle des départements. Par exemple, dans le Limousin, où le taux de pacsés (83,8 signataires pour 10000 habitants de 18 ans et plus fin 2005) est très proche de la moyenne nationale, il est maximal dans l'arrondissement de Limoges (122), le seul situé audessus de la moyenne régionale. Loin derrière se situent les autres arrondissements : ceux de la Creuse et celui de Bellac en Haute-Vienne ont des taux compris entre 37 et 43 . Le poids de la population rurale et la structure par âges pourraient expliquer en partie un tel déséquilibre. Celui-ci était d'ailleurs encore plus accentué aux débuts du pacs. L'arrondissement de Limoges abritait à lui seul $63 \%$ des pacsés du Limousin en 1999 contre $57 \%$ en 2005, et certains tribunaux d'instance d'arrondissements ruraux comme celui de Bourganeuf n'avaient 
encore enregistré aucune signature fin 1999. Ceci montre que la capitale régionale a joué la fonction de relais diffuseur.

Fig. 5 : Poids des capitales régionales et de leur département dans la répartition des signataires de pacs fin 2005

\begin{tabular}{|c|c|c|c|}
\hline & \multicolumn{2}{|c|}{$\begin{array}{c}\text { Part du département } \\
\text { de la capitale régionale (en \%) }\end{array}$} & \multirow[b]{2}{*}{ Ecart (en pts) } \\
\hline & $\begin{array}{c}\text { dans le nombre } \\
\text { de pacsés de } \\
\text { la région }\end{array}$ & $\begin{array}{c}\text { dans la population } \\
\text { de la région } \\
\text { de } 18 \text { ans et + }\end{array}$ & \\
\hline Ile-de-France & 37,4 & 21,0 & 16,4 \\
\hline Auvergne & 60,8 & 46,1 & 14,7 \\
\hline Limousin & 63,4 & 49,6 & 13,8 \\
\hline Midi-Pyrénées & 52,4 & 40,6 & 11,8 \\
\hline Bourgogne & 42,9 & 31,3 & 11,6 \\
\hline Basse-Normandie & 56,5 & 45,5 & 11,0 \\
\hline Franche-Comté & 55,1 & 44,5 & 10,6 \\
\hline Champagne-Ardenne & 52,5 & 42,3 & 10,2 \\
\hline Languedoc-Roussillon & 48,5 & 39,1 & 9,4 \\
\hline Bretagne & 38,2 & 29,5 & 8,7 \\
\hline Pays-de-la-Loire & 43,7 & 35,1 & 8,6 \\
\hline Poitou-Charentes & 32,1 & 24,4 & 7,7 \\
\hline Alsace & 66,7 & 59,4 & 7,3 \\
\hline Centre & 31,1 & 25,0 & 6,1 \\
\hline Corse & 50,5 & 45,2 & 5,3 \\
\hline Haute-Normandie & 75,0 & 69,9 & 5,1 \\
\hline Aquitaine & 48,9 & 43,9 & 5,0 \\
\hline Provence-Alpes-Côte-d'Azur & 44,2 & 40,4 & 3,8 \\
\hline Rhône-Alpes & 30,3 & 28,0 & 2,3 \\
\hline Nord-Pas-de-Calais & 66,1 & 63,9 & 2,2 \\
\hline Picardie & 31,9 & 30,5 & 1,4 \\
\hline Lorraine & 38,9 & 44,2 & $-5,3$ \\
\hline
\end{tabular}

Il apparaît donc clairement que le pacs trouve un terrain favorable dans les capitales régionales, quelle que soit leur taille. Ainsi, 10 des 12 arrondissements de province les plus favorables au pacs contiennent une capitale régionale. Ce sont généralement les espaces les plus peuplés et urbanisés. Aussi, le pacs constitue actuellement une innovation sociale davantage adoptée par les villes, que l'on peut qualifier de foyers émetteurs. Mais son taux n'est pas lié de manière systématique à celui de l'urbanisation : le coefficient de corrélation entre les taux d'urbanisation et de pacsés n'est que de 0,44. En effet, l'observation des résidus de régression (Figure 6) montre des départements très urbanisés qui s'écartent de cette relation (Nord-Pas-de-Calais, banlieue parisienne). Parmi les départements de tête, là où le taux de pacs est supérieur à ce que le taux d'urbanisation laisserait prévoir, beaucoup abritent des villes qui ne sont pas de grandes métropoles. Mais il s'agit toujours de capitales régionales ou de pôles universitaires (Ruelland, 2006) ou les deux : Poitiers, Nancy, Montpellier, Besançon, Clermont-Ferrand, Limoges, Rennes, Dijon, Grenoble. Dans le cas exemplaire de Poitiers, le taux de pacs du département de la Vienne est à la fin de 2005 supérieur de 24 points à celui des Deux-Sèvres, de 22 points à celui de la Charente et même de 10 points à celui de la Charente-Maritime, départements commandés pourtant par des agglomérations de taille assez comparable à celle de Poitiers. 
Fig. 6 : Écarts à la relation linéaire entre le taux d'urbanisation et le taux de pacs

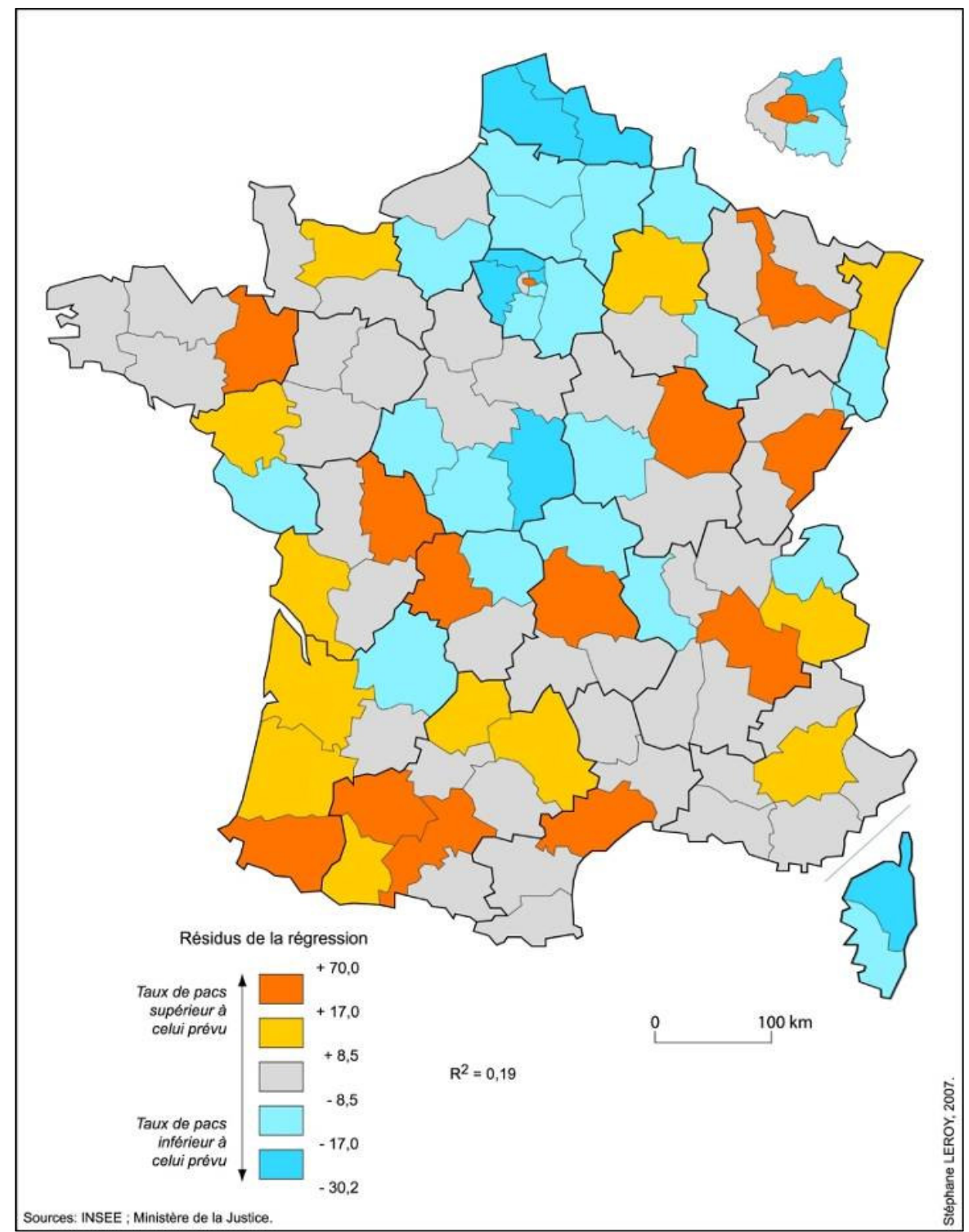

L'hypothèse d'une relation entre la surreprésentation des fonctionnaires, des actifs les plus qualifiés et celle des pacsés est vérifiée. La valeur la plus élevée des coefficients de corrélation entre le taux de pacs et la part de chacune des 42 professions et catégories sociales (niveau 3) est celle de la catégorie «professeurs, professions scientifiques » qui est égale à 0,82 (à l'opposé le coefficient est de $-0,31$ pour les exploitants agricoles et de $-0,47$ pour les ouvriers). Elle explique l'essentiel des variations géographiques du taux de pacs (Figure 7). La part du secteur public est comprise entre 32 et $42 \%$ des actifs à Poitiers, Limoges, Bordeaux, Toulouse, Montpellier, Clermont-Ferrand et Nancy, soit une proportion supérieure à celle de Lyon, Marseille ou Nice. Si les coefficients de corrélation pour les professions très qualifiées du secteur privé sont positifs et assez forts, ils sont toujours inférieurs à ceux de leurs homologues du secteur public $(0,43$ pour les cadres du secteur privé contre 0,55 pour ceux de la Fonction Publique). Si nous ne pouvons pas affirmer que ce sont les professeurs et scientifiques qui se pacsent, il semble tout de même qu'un élément de motivation de la 
signature de pacs est le bonus de points qu'il apporte pour les demandes de mutation dans la fonction publique ${ }^{5}$. La saisonnalité du pacs en est même dépendante. Mais la répartition des enseignants du secondaire étant plus homogène (dépendante de la population scolarisée) que celle des universitaires, on peut raisonnablement penser que ces derniers contribuent davantage aux écarts entre villes et départements. Poitiers, premier arrondissement de province avec un taux de pacsés de 164 pour 10000 habitants de 18 ans et plus est aussi l'aire urbaine présentant la plus forte densité d'étudiants (Julien et al., 2001). Nous voulons insister sur le fait que cette hypothèse de l'impact des mutations au sein de la Fonction Publique dans l'explication des différences départementales de taux de pacs est à nuancer. Ainsi, la part des autres catégories de la Fonction Publique dans la population des départements est faiblement ou aucunement corrélée avec le taux de pacs (-0,06 pour les agents de service de la Fonction Publique par exemple).

Fig. 7 : Écarts à la relation linéaire entre la part des professeurs et professions scientifiques et le taux de pacs

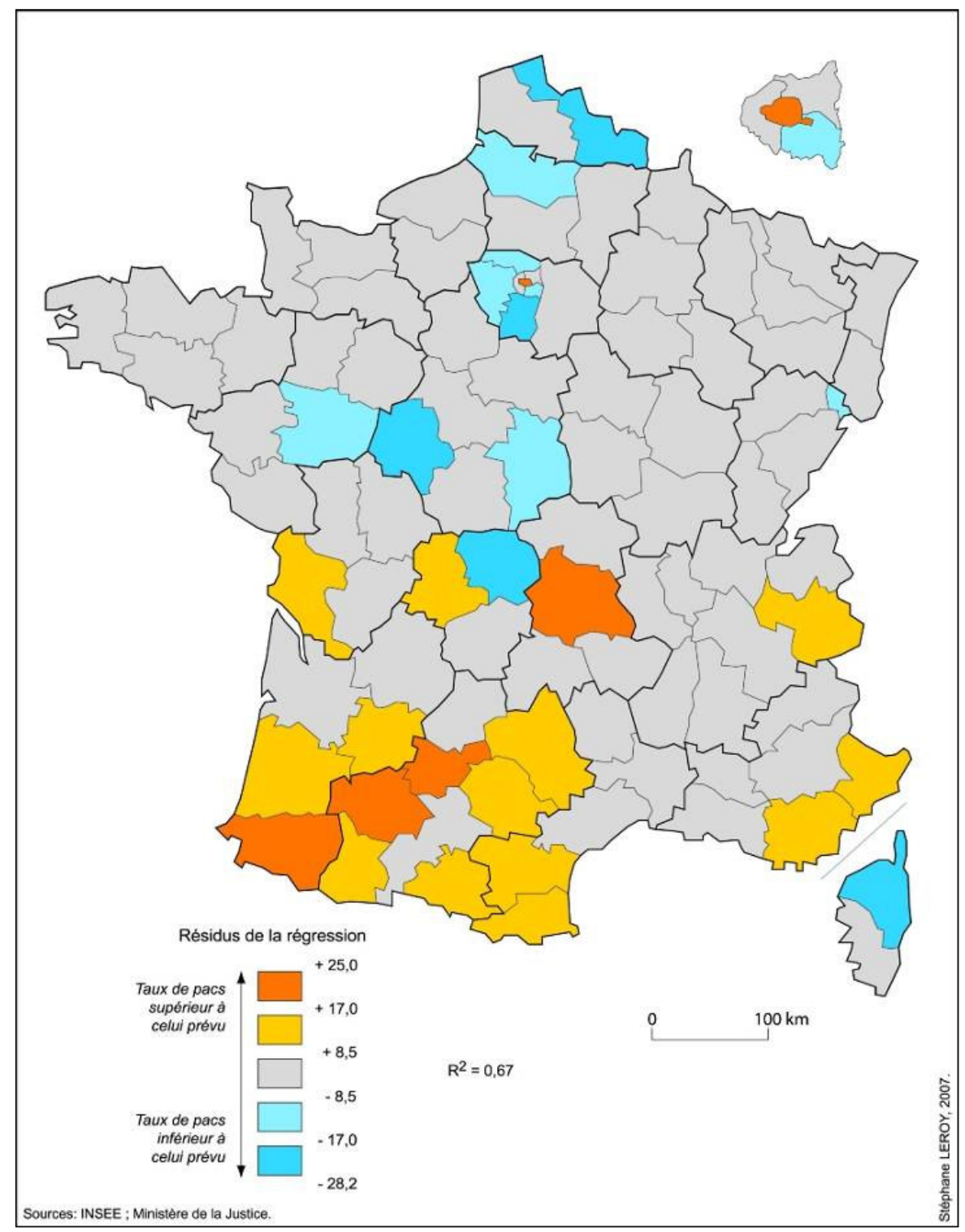




\subsection{Paris, capitale du pacs}

Un dernier trait de la géographie régionale du pacs est la place privilégiée de Paris. Celle-ci relève d'un double clivage, à l'échelle nationale et francilienne. Certes, en l'absence de données statistiques disponibles sur d'autres communes de France, on ne peut comparer le cas de Paris qu'avec celui d'autres départements ; une confrontation avec d'autres villes-phares du pacs aurait été fort instructive, mais reste impossible pour l'instant. Le département de Paris est en tout cas celui des records pour le pacs. Cumulant plus de 16000 signatures au 31 décembre 2005, la capitale présente une très importante concentration de pacsés. Avec un taux de près de 174 signataires pour 10000 habitants de 18 ans et plus, Paris est en effet loin devant le deuxième département de France (Haute-Garonne, 127). Le nombre d'enregistrements de pacs dans la ville de Paris pèse pour plus de $37 \%$ dans ceux de Île-deFrance (alors que la population de 18 ans et plus n'en représente que $21 \%$ ) et équivaut presque à celui de la région Provence-Alpes-Côte d'Azur.

La primauté parisienne était encore plus flagrante au début du pacs. Fin 1999, Paris (avec près de 900 contrats enregistrés en 6 semaines) représentait 14,4\% des signatures totales de la France pour seulement 3,3\% de la population; fin 2005, la proportion a été divisée par deux pour s'établir un peu au-dessus de $7 \%$. On retrouve ici le processus de diffusion du pacs, des terres d'élection, les capitales nationale et régionales, qui ont joué un rôle de fer de lance, aux terres de mission. Le contraste initial de la géographie du pacs perdure, mais de façon peu à peu édulcorée.

Si Île-de-France n'est pas la région la plus favorable au pacs, c'est à la tiédeur de la banlieue qu'elle le doit. Le découpage administratif particulier de Île-de-France, avec une aire urbaine subdivisée en plusieurs départements, permet de mettre en évidence l'opposition entre une ville-centre très réceptive à l'innovation que représente le pacs et une périphérie plus rétive, même si la dynamique récente montre un rattrapage relatif de celle-ci, à l'exception du département de la Seine-Saint-Denis. Dans les trois départements de la petite couronne, le taux de pacsés est plus de deux fois inférieur à celui de Paris $(85,4)$ fin 2005 , soit une valeur inférieure à la moyenne de la France métropolitaine et équivalente par exemple à celle des Hautes-Pyrénées, département nettement moins urbanisé. Dans les quatre départements de la grande couronne, le taux tombe à 70,4, soit une valeur inférieure à celle de la Corrèze. Nous avons la confirmation que la géographie du pacs ne saurait être réduite à une simple opposition ville-campagne.

Il est intéressant de signaler que les résidus des régressions entre les professions et catégories sociales, même supérieures, et le taux de pacs sont toujours fortement positifs pour Paris, à l'exception de la catégorie " professions de l'information, des arts et des spectacles », catégorie extrêmement concentrée dans la capitale. Certes, Paris valide les facteurs explicatifs relevés précédemment. Sa concentration élevée de pacsés confirme l'existence d'une relation statistique entre la proportion d'emplois qualifiés ou métropolitains supérieurs (Julien, 2002), voire entre ce que Richard Florida (2002) définit comme la «classe créative », et le taux de pacs. Mais ce profil socioprofessionnel est insuffisant pour rendre compte de la 
surreprésentation des signataires de pacs à Paris. D'ailleurs les arrondissements parisiens où les cadres et professions intellectuelles supérieures sont les plus nombreux (ouest et rive gauche) sont curieusement ceux où l'on se pacse le moins. Les écarts à l'intérieur de Paris sont encore plus importants qu'aux autres échelles étudiées dans cet article, témoignant de la vigueur de la fragmentation sociale dans la capitale : ainsi le taux le plus faible, 60 dans le $\mathrm{XVI}^{\mathrm{e}}$ arrondissement, est plus de 6 fois inférieur au taux le plus fort, 371 dans le $\mathrm{II}^{\mathrm{e}}$ arrondissement. Celui-ci est considéré comme l'archétype de l' «arrondissement yuppie », suivant l'expression utilisée par Alfred Dittgen (2005), où les jeunes adultes sont surreprésentés, en particulier les «néo-bourgeois » (Pinçon et Pinçon-Charlot, 2004), plus réceptifs aux innovations sociales contemporaines que la bourgeoisie traditionnelle. L'attitude par rapport au pacs ne renvoie pas seulement à un profil socioéconomique type. Elle est peutêtre à relier à l'adhésion à un système de valeurs que Richard Florida (2002) regroupe sous le terme général de «tolérance ».

\section{Les logiques complexes de l'appropriation et de la diffusion du pacs}

La complexité des structures géographiques du pacs, à toutes les échelles, renvoie au jeu de facteurs multiples, pour certains non quantifiables, ayant une simple valeur heuristique ou provisoirement impossibles à évaluer. Ainsi des facteurs culturels qui expliquent certainement en partie les contrastes de sa diffusion.

\subsection{Des facteurs multiples pour une géographie provisoire}

Si l'opposition entre centre et périphérie constitue l'articulation majeure de la géographie du pacs aux échelles nationale et régionale, il n'y a pas un facteur social, culturel ou économique unique qui s'applique à toutes les échelles et pour tous les espaces, même si la corrélation entre surreprésentation des enseignants et des professions scientifiques et taux de pacs élevés est très forte. Le clivage urbain/rural est clair à l'échelle régionale, beaucoup moins à l'échelle nationale où la banlieue parisienne et les départements ruraux du sud font jeu égal ; MidiPyrénées, la première région du pacs, est largement plus rurale que le Nord-Pas-de-Calais qui se retrouve dans les bonnes dernières.

On trouve aussi des régions à population plus jeune que la moyenne nationale qui plébiscitent le pacs (Île-de-France) et d'autres qui le boudent (Nord-Pas-de-Calais et régions du nord du Bassin Parisien). Pour les départements, le coefficient de corrélation entre la part des 20-29 ans dans la population et le taux de pacsés est égal à 0,58, valeur assez élevée. Toutefois, la géographie des résidus de régression montre un net clivage entre le sud et le nord (Figure 8). Globalement, on se pacse plus que ne le laisserait supposer la structure par âge au sud (et à Paris) et c'est le contraire dans beaucoup de départements septentrionaux (en particulier ceux du Nord-Pas-de-Calais). Des départements ayant la même proportion d'adultes âgés de 20 à 29 ans ont des taux de pacsés très dissemblables : ainsi de l'Hérault (113) et de la Somme (70). Comme les autres indicateurs sociodémographiques, la structure par âge ne peut rendre compte à elle seule des disparités spatiales du pacs. 
Fig. 8 : Écarts à la relation linéaire entre la part des 20-29 ans et le taux de pacs

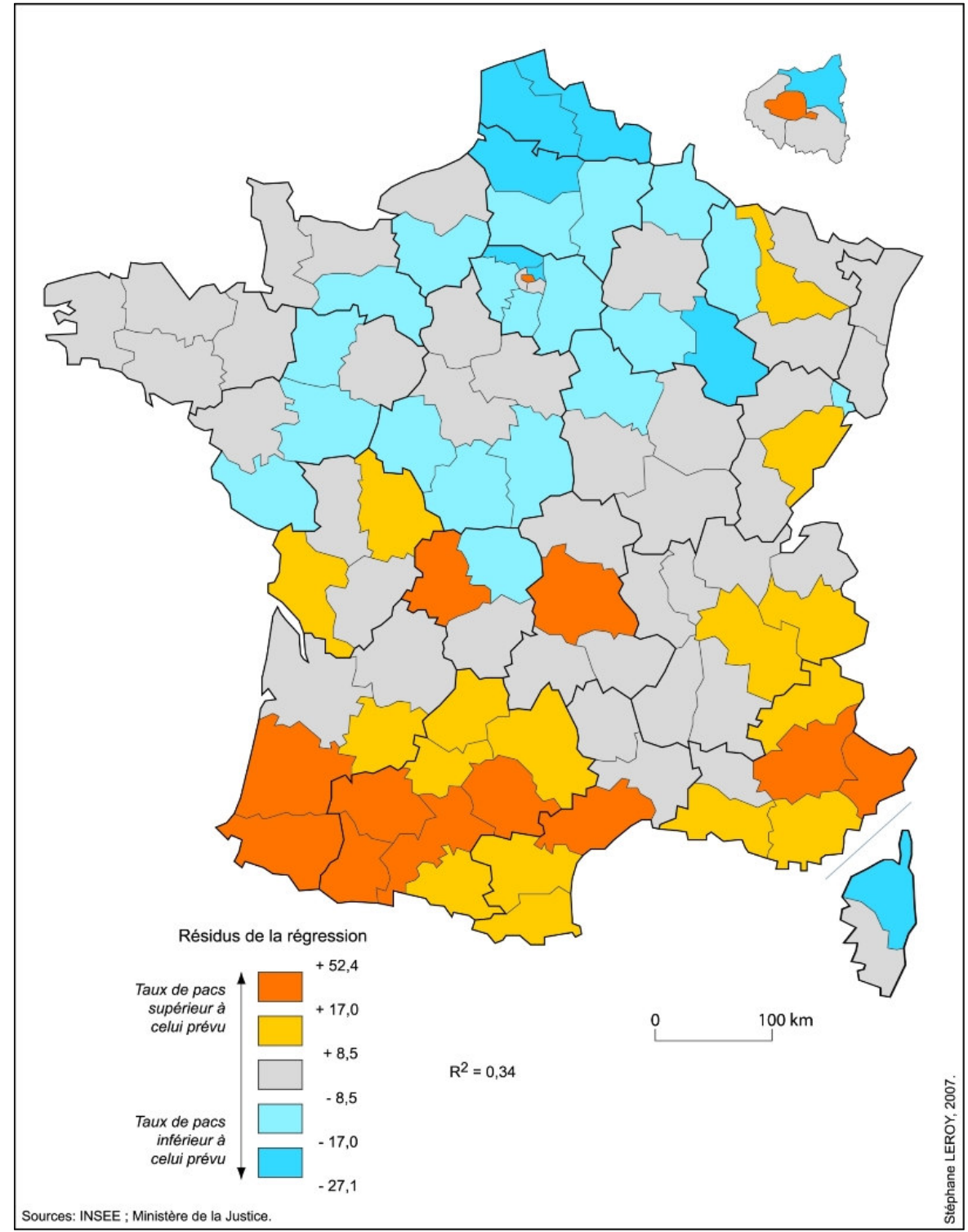

Comme nous l'avons suggéré pour Paris, il est probable que de nombreux traits culturels, difficiles à identifier, isoler et mesurer, du local au régional, participent des variations géographiques et de la diffusion spatiale du pacs : ainsi des traditions politiques, des pratiques religieuses, des modèles familiaux, des modes de vie, etc. qui contribuent sans doute à l'explication de l'appropriation différenciée dans le temps et dans l'espace de cette innovation sociale. De même, nous ne pouvons pas évaluer l'impact de la répartition de la population homosexuelle sur la géographie du pacs. Il est cependant attendu que ce groupe, «mécaniquement» surreprésenté dans le pacs, participe et même renforce certaines dynamiques spatiales évoquées précédemment: migration vers Île-de-France ou le Midi à l'âge adulte, à l'occasion d'une décohabitation de la cellule familiale et de la région d'origine (Eribon, 1999) ; métropolisation, déjà ancienne (Aldrich, 2004), et accentuation de la centralité, avec un afflux principalement vers Paris et certaines capitales régionales ${ }^{6}$, où les 
homosexuels, notamment les plus jeunes, savent qu'ils trouveront à la fois l'anonymat et la visibilité, presque impossibles ailleurs (Leroy, 2005).

$\mathrm{Si}$ les variations géographiques du pacs sont maintenant bien connues, on continue de se heurter à l'imprécision des données sur les pacsés et à la difficulté de saisir et de mesurer les facteurs d'ordre culturel. L'analyse de la dynamique de la géographie du pacs depuis sa création permet toutefois d'approcher les logiques de la diffusion de cette innovation.

\subsection{De la diffusion spatiale à la diffusion sociale du pacs ?}

L'observation de l'évolution du poids relatif de chaque département dans le total des pacsés, si elle ne permet pas d'apporter des réponses claires à la géographie complexe du pacs, est tout de même significative. La population de pacsés a augmenté partout mais avec des rythmes différents. La principale opposition concerne Paris (seule dans la classe A) par rapport au reste de la France (Figure 9).

La surreprésentation des signataires à Paris dans les premières semaines du pacs, pointée précédemment, est donc très forte. C'est bien au moment de la mise en place de ce nouveau contrat d'union que les écarts ont été les plus importants. Généralement, l'innovation est d'abord adoptée par une minorité infime, située au sommet de la hiérarchie sociale, c'està-dire par une population qui possède un niveau d'éducation, des ressources financières et un accès à l'information supérieurs à la moyenne (Rogers, 1983). Il y avait à l'évidence une attente importante dans la population parisienne. Peut-on l'expliquer par la forte concentration de jeunes actifs qualifiés, de la «nouvelle classe moyenne supérieure » attirée par les villescentres (David Ley parle en 1996 de new middle class) et par celle, impossible à mesurer mais fortement supposée, de la population homosexuelle (Eribon, 1999 ; Leroy, 2005), beaucoup plus encline à afficher son identité et son «droit de centralité » dans la capitale qu'ailleurs ? A partir de 2000, le poids relatif de Paris ne cesse de diminuer : l'innovation sociale se diffuse. Un autre trait de l'évolution d'ensemble est l'opposition entre les départements qui ont connu un afflux relativement important de signataires dès l'entrée en vigueur de la loi en 1999 (classes B1 et B2) et ceux pour lesquels le démarrage a été plus faible et surtout décalé dans le temps (classes $\mathrm{C} 1$ et $\mathrm{C} 2$ ).

L'observation de la répartition spatiale de ces différentes classes ouvre quelques pistes supplémentaires à la compréhension de la géographie du pacs. Aucun des facteurs explicatifs de la répartition des pacsés à la fin de l'année 2005, relevés précédemment, ne suffit à rendre compte des évolutions relatives. Bien sûr, une France coupée entre une moitié Nord-Est (beaucoup de départements dans les classes B) et une moitié Sud-Ouest (beaucoup de départements dans la classe C2) se devine, mais avec de nombreuses exceptions. De même, si le pacs s'est diffusé du centre parisien vers les centres périphériques (11 départements de capitale régionale dans la classe $\mathrm{C} 2$ et 6 dans la classe $\mathrm{C} 1$ ), puis vers les marges, cette diffusion par sauts ne suit pas complètement les canaux hiérarchiques habituels de la plupart des innovations (Saint-Julien, 1995). C'est donc bien l'image du kaléidoscope qui correspond le mieux aux dynamiques spatio-temporelles du pacs. 
Fig. 9 : Évolution relative du nombre de pacsés par département entre 1999 et 2005

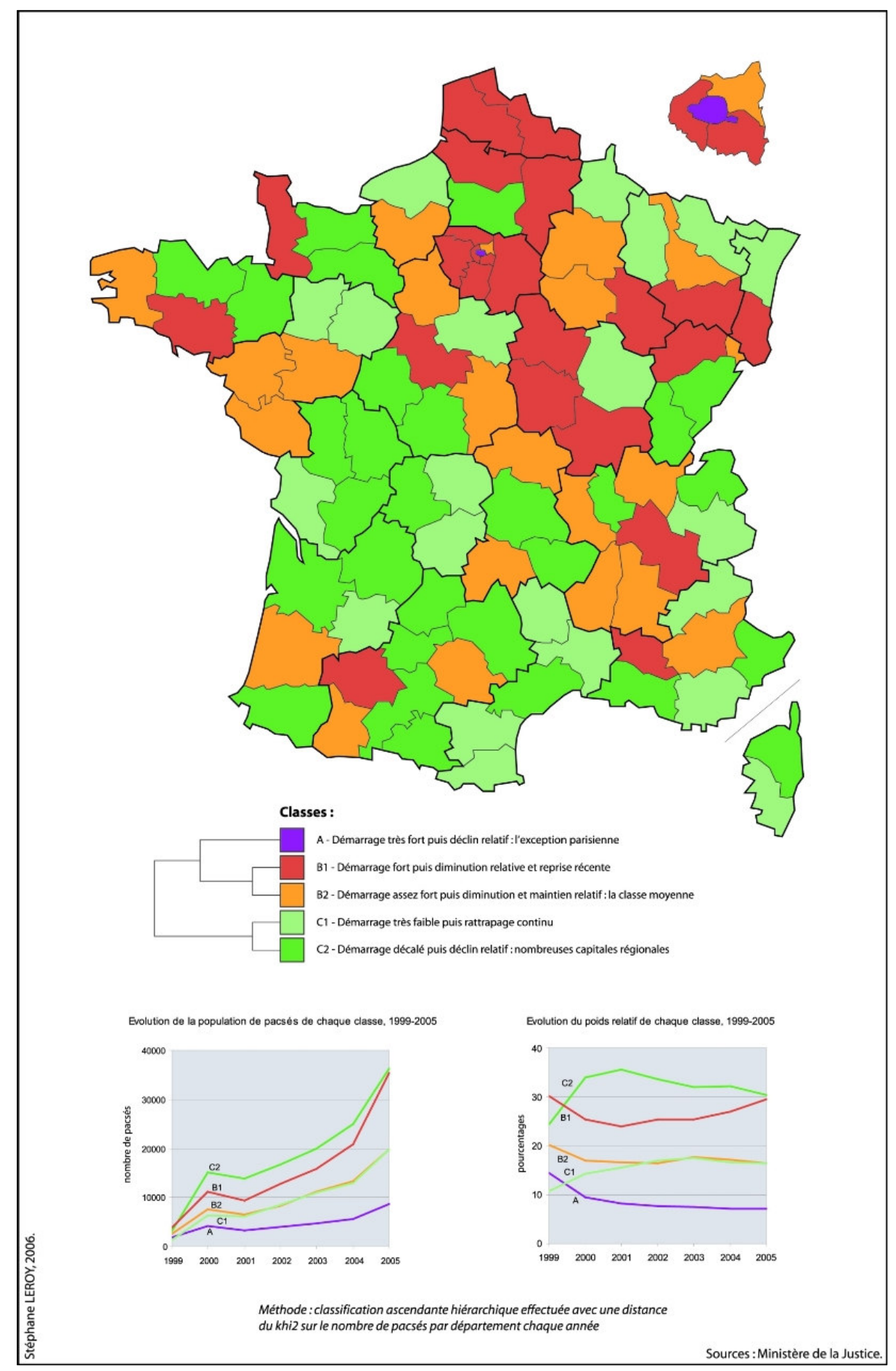

Cette diffusion spatiale complexe n'est-elle pas combinée à une diffusion sociale, plus ou moins rapide, des couples de même sexe aux couples de sexe opposé ? On a déjà évoqué la surreprésentation probable des couples de même sexe la première année d'existence des contrats d'union (Festy, 2006). Le relais hétérosexuel pour les signatures de pacs aurait été d'autant plus tardif que l'attachement au mariage reste fort. Dans de nombreuses régions où le pacs a rapidement décliné avant de reprendre (classes B), le ratio mariage/pacs est particulièrement élevé (Nord-Pas-de-Calais, Picardie, Pays de la Loire, Bourgogne); ici 
l'adoption du pacs par les couples hétérosexuels fut sans doute plus lente. Corse, Centre et Basse-Normandie échappent à ce schéma (à l'échelle départementale, la corrélation entre le taux de mariage et celui de pacs, calculés sur la période 2000-04, est faible). A l'inverse, les régions les plus favorables au pacs (Midi-Pyrénées, Languedoc-Roussillon, Aquitaine, Limousin notamment), caractérisées par un ratio mariage/pacs faible, n'ont pas connu de diminution relative dans les 2 années qui suivent la naissance du pacs (classes C). Ici les couples de sexe opposé ont rapidement dû adopter le pacs et prendre le relais des couples de même sexe dans les greffes des tribunaux d'instance. La diffusion sociale aurait donc adopté des rythmes différents en fonction des spécificités sociodémographiques, économiques et surtout culturelles des territoires.

\section{Conclusion}

Nous avons montré la complexité de la géographie du pacs, témoignant de la grande hétérogénéité de la société et de ses dynamiques. La France enthousiaste face au pacs occupe un espace réduit, celui des capitales, nationale ou régionales, des villes universitaires, des régions attractives pour les flux migratoires de jeunes actifs. Ces espaces ouverts à l'innovation sociale sont globalement plus méridionaux que septentrionaux. La France encore rétive est plus étendue ; elle juxtapose des espaces dans lesquels les réticences face au pacs ont des explications sans doute très variées et qui peuvent se combiner : sociodémographiques, économiques, culturelles, etc. Ainsi, il nous semble que le pacs, même si ses signataires ne représentent encore qu'une faible part de la population, apparaît comme une innovation dont la diffusion est révélatrice de la diversité sociale de la France, et même de la fragmentation sociospatiale, à l'œuvre à différentes échelles. Des données à une échelle plus grande permettraient d'affiner ces observations. Ainsi, par exemple, il serait particulièrement intéressant pour la géographie sociale urbaine de savoir si les fortes disparités relevées à l'intérieur de Paris comme entre celle-ci et sa banlieue se retrouvent dans d'autres aires urbaines.

Nous nous sommes risqués «en terrain découvert »(Collignon et Staszak, 2004, p. 39) en faisant le pari de défricher la géographie du pacs, pressentant son grand intérêt pour la compréhension de la géographie sociale de la France contemporaine mais conscients, aussi bien des limites de l'approche quantitative que de celles imposées par l'imprécision des données, contrairement à la situation dans d'autres pays. Patrick Festy le souligne : «il est dommage qu'en France la statistique soit empêchée de fournir un détail minimum sur le type de pacs et le sexe des partenaires. Cette restriction est difficilement compréhensible, quand on connaît le large éventail de statistiques anonymes et exhaustives disponibles en France sur des sujets tout aussi sensibles (avortement, causes de décès, détentions, illettrisme, etc.), qui n’ont jamais donné lieu au moindre dévoilement de la vie privée, grâce aux dispositifs de sécurité légalement mis en œuvre. On se prive ainsi d'un instrument important d'évaluation des effets de la loi. On en est réduit à des conjectures qui n'auront jamais la pertinence d'une mesure 
précise des comportements » (2001, p. 4).

Les enjeux sont nombreux à essayer d'interroger l'espace avec de nouvelles grilles de lecture. A propos de l'interdiction de divulguer le sexe des signataires de pacs, la CNIL confessait dans son Rapport d'activité pour 1999 : «Sans doute une telle précaution pourra-telle perdre de sa justification au fur et à mesure de l'évolution des mœurs » (Bloche et Michel, 2001). Le chercheur doit se réjouir qu'aujourd'hui en 2007, cette «précaution » ait été jugée superflue. Elle ouvre de nouvelles perspectives, d'abord pour préciser l'explication des variations spatiales du pacs, ensuite pour élaborer une géographie des différents types d'unions hétérosexuelles, enfin pour contribuer à asseoir une géographie naissante des homosexuels en France.

\section{Bibliographie}

Aldrich R. (2004), «Homosexuality and the City : An Historical Overview », Urban Studies, vol.41, n 9 , p. 1719-1737.

Bloche P. et Michel J.-P. (2001), Rapport d'information déposé par les commissions des affaires culturelles et des lois, sur l'application de la loi n'99-944 du 15 novembre 1999 relative au pacte civil de solidarité, 13.11.2001, Paris, $\mathrm{n}^{\circ} 3383$, http://www.assembleenationale.fr.

Borrillo D. et Fassin E. (2004), «The Pacs, Four Years Later : A Beginning or an End ? », in M. Digoix et P. Festy (ed.), Same-sex couples, same-sex partnerships and homosexual marriages. A Focus on cross national differentials, Paris, Documents de travail de l'INED, n¹24, p. 19-25.

Chayet D. (2006), «Les hétérosexuels, premiers utilisateurs du pacs », Le Figaro, 15.11.2006, p. 11.

Collignon B. et Staszak J.-F. (2004), «Que faire de la géographie postmoderniste ? », L'Espace géographique, $\mathrm{n}^{\circ} 1$, p. 38-41.

Coulmont B. (2003), «Géographie de l'Union Civile au Vermont», Mappemonde, n71, p. $13-18$.

De Singly F. (2000), « Sur la crise de la vie conjugale », in Y. Michaud (dir.), Qu'est-ce que la société ?, Université de tous les savoirs, vol. 3, Paris, Odile Jacob, p. 481-491.

Digoix M. et Festy P. (ed.) (2004), Same-sex couples, same-sex partnerships and homosexual marriages. A Focus on cross-national differentials, Paris, Documents de travail de l'INED, $\mathrm{n}^{\circ} 124$.

Dittgen A. (2005), « Paris, une démographie atypique », Population \& Avenir, n671, p. 4-7.

Eribon D. (1999), Réflexions sur la question gay, Paris, Fayard.

Eribon D. (2000), Papiers d'identité. Interventions sur la question gay, Paris, Fayard.

Eribon D. (dir.) (2003), Dictionnaire des cultures gays et lesbiennes, Paris, Larousse.

Festy P. (2001), «Pacs : l'impossible bilan », Population et Sociétés, n³69, p. 1-4.

Festy P. (2006), «Légaliser les unions homosexuelles en Europe : innovations et paradoxes », Population et Sociétés, $\mathrm{n}^{\circ} 424$, p. 1-4.

Florida R. (2002), The rise of the creative class. And how it's transforming work, leisure, community and everyday life, New York, Basic Books.

Gates G.J. et Ost J. (2004), The Gay and Lesbian Atlas, Washington D.C., The Urban Institute Press.

Héritier F. (2000), «Anthropologie de la famille », in Y. Michaud (dir.), Qu'est-ce que la société ?, Université de tous les savoirs, vol. 3, Paris, Odile Jacob, p. 467-480. 
Holtz M. (2000), «La spectaculaire multiplication des Pacs », Libération, 21.01.2000, p. 17.

Julien P. (2002), « Onze fonctions pour qualifier les grandes villes », INSEE Première, $\mathrm{n}^{\circ} 840$.

Julien P., Laganier J. et Pougnard J. (2001), «Les études supérieures: un motif de migration $»$, INSEE Première, $n^{\circ} 813$.

Kosofsky Sedgwick E. (1990), Epistemology of the closet, Berkeley, University of California Press.

Léon O. et Godefroy P. (2006), «Les échanges de population entre zones d'emploi. Six profils types », INSEE Première, $\mathrm{n}^{\circ} 1074$.

Leroy S. (2005), «Le Paris gay. Eléments pour une géographie de l'homosexualité », Annales de Géographie, $\mathrm{n}^{\circ} 646, \mathrm{p} .579-601$.

Ley D. (1996), The new middle class and the remaking of the central city, Oxford, Oxford University Press.

Offner J.-M. (2003), "Innovation », in J. Lévy, M. Lussault (dir.), Dictionnaire de la géographie et de l'espace des sociétés, Paris, Belin, p. 513.

Pinçon M. et Pinçon-Charlot M. (2004), Sociologie de Paris, Paris, La Découverte, coll. « Repères ».

Putnam R. (2000), Bowling alone. The collapse and revival of American community, New York, Simon \& Schuster.

Prioux F. (2006), «Vivre en couple, se marier, se séparer : contrastes européens », Population et Sociétés, $\mathrm{n}^{\circ} 422$, p. 1-4.

Rault W. (2004), « The best way to court. The French mode of registration and its impact on the social significance of partnerships », in M. Digoix et P. Festy (ed.), Same-sex couples, same-sex partnerships and homosexual marriages. A Focus on cross-national differentials, Paris, Documents de travail de l'INED, n²124, p. 27-33.

Richet-Mastain L. (2006), «Bilan démographique 2005 - En France, la fécondité des femmes augmente toujours », INSEE Première, $\mathrm{n}^{\circ} 1059$.

Richet-Mastain L. (2007), «Bilan démographique 2006 : un excédent naturel record », INSEE Première, $\mathrm{n}^{\circ} 1118$.

Rogers E.M. (1983), Diffusion of innovations, New York, Free Press.

Ruelland N. (2006), «Le pacte civil de solidarité : importante progression en 2005 », Infostat Justice, $\mathrm{n}^{\circ} 89$, p. 1-4.

Ruelland N. et Tonnerre M. (2003), «Trois années d'application du pacte civil de solidarité », Infostat Justice, $\mathrm{n}^{\circ} 64$, p. 1-4.

Saint-Julien T. (1995), «Diffusion spatiale», in A. Bailly, R. Ferras et D. Pumain (dir.), Encyclopédie de géographie, Paris, Economica, p. 559-581.

Schiltz M.-A. (2004), «Pacs: The chaotic emergence of the category in French social surveys », in M. Digoix et P. Festy (ed.), Same-sex couples, same-sex partnerships and homosexual marriages. A Focus on cross-national differentials, Paris, Documents de travail de l'INED, n $^{\circ} 124$, p. 225-232.

\footnotetext{
${ }^{1}$ Pour être tout à fait exact, il faut ôter à ce total cumulé les 26713 dissolutions prononcées entre le 15 novembre 1999 et le 31 décembre 2005.

${ }^{2}$ Sur l'expérience du «placard » et les conséquences de sa sortie, on se reportera à : Kosofsky Sedgwick (1990).

3 « 274400 mariages ont été célébrés en 2006, soit 8800 de moins qu'en 2005. La hausse de 2005 apparait donc comme une exception et la diminution observée entre 2001 et 2004 reprend. Le nombre de mariages est à son plus bas niveau depuis $1995 »$ (Richet-Mastain, 2007).
} 
${ }^{4}$ L'ensemble des données sur les professions et catégories socioprofessionnelles est issu du tableau du recensement de la population de 1999 MEN1 détaillé qui donne la répartition en 42 postes de la population des ménages ordinaires. Tous les membres d'un ménage sont comptabilisés dans le poste de la personne de référence. Il s'agit de l'exploitation au quart du recensement.

${ }^{5}$ En 2001, le magazine Le Point a fait état d'une «vague de pacs blancs chez les profs » $\left(\mathrm{n}^{\circ} 1484,23 / 02 / 2001\right)$, les estimant à 4000 , en se gardant bien de préciser la source de ce chiffre évidemment invérifiable. On trouve aussi sur le web des annonces d'étudiants d'IUFM du sud de la France, recherchant des signataires de «pacs blanc » habitant leur région, afin de limiter voire d'éviter le risque d'une affectation trop septentrionale.

${ }^{6}$ Au premier rang desquelles figure Montpellier, désignée à l'issu d'un sondage pour le magazine gay et lesbien Têtu (n ${ }^{\circ} 79$, juin 2003), comme la ville de province la plus gay friendly, c'est-à-dire la plus accueillante et ouverte à la population homosexuelle. 